\title{
Structure, Dielectric Properties and AC Behavior of Commercial Polytetrafluroethylene (PTFE) Polymer
}

\author{
Shujahadeen B. Aziz and Hameed M. Ahmed \\ Department of Physics, Faculty of Science and Science Education, University of Sulaimani \\ Kurdistan Region - Iraq
}

\begin{abstract}
In this work the relative permittivity $\left(\varepsilon^{\prime}\right)$, dielectric loss $\left(\varepsilon^{\prime \prime}\right)$ and AC-conductivity $\left(\sigma_{A C}\right)$ for commercial polytetrafluroethylene (PTFE) have been measured at different temperatures $\left(20-110^{\circ} \mathrm{C}\right)$ and over the frequency range from 10 KHz to $1 \mathrm{MHz}$. The infrared (IR) spectra of PTFE also been investigated to detect the presence of polar groups and carbon double bonds. The relative permittivity had observed to decrease with increasing frequency and temperature. Some loss peaks were observed in the dielectric loss spectra, which referred to the relaxation, arises from the orientation of unsaturated double $(C=C)$ bond and polar additives. The diameter of the semicircles in Cole-Cole $\left(\varepsilon^{\prime \prime}\right.$ versus $\left.\varepsilon^{\prime}\right)$ plots are not coincides with $\mathrm{x}$-axis at different temperature which reveals that the relaxation processes are non-Debye type. The AC-conductivity increases with increasing of frequency and almost independent on the temperature.
\end{abstract}

Index Terms-AC conductivity, Dielectric loss, IR spectra, non-polar PTFE, relative permittivity.

\section{INTRODUCTION}

Polymers are large molecules formed by linking together of many small molecular units know as monomers. These are covalently bonded together in any conceivable pattern. Molecular structure and the arrangement of polymer chain in a solid are important factor in determination their properties. Polymers are ideal material for industrial application because they have desirable properties such as durability, processability, transparency, electrical and thermal resistance. Indeed, we live in the world of polymers. That is why scientist and technologists have termed this area as the polymeric age (Singh, 2006). Dielectric measurements such as relative

ARO-The Scientific Journal of Koya University

Volume 2013, Article ID, 5 pages

DOI: $10.14500 /$ aro. 10033

Received 08 October 2013; Accepted 03 November 2013

Regular research paper: Published 03 December 2013

Corresponding author's e-mail: shujaadeen78@yahoo.com

Copyright () 2013 Shujahadeen B. Aziz and Hameed M. Ahmed.

This is an open access article distributed under the Creative Commons Attribution License. permittivity and dielectric loss reveal significant information about the chemical and physical state of polymers (Vijayalakshmi, Ashokan and Shridhar, 2000). For electrical insulation application, a large band gap, a low relative permittivity and a low dielectric loss over a wide frequency range are desirable (Dilip, 1994). Non-polar polymers like polyethylene (PE), polypropylene (PP) and polytetrafluoroethylene (PTFE) are very important electrical insulating and dielectric materials. They are used in the increasingly high AC electric field strength region approaching to the limit of electrical breakdown strength of the material (Fujii, et al., 2006). PTFE polymer is an engineering thermoplastic which shows a remarkable chemical resistance, electrical insulating properties as well as a low friction coefficient. These mentioned properties enables PTFE polymer as a raw material for non-stick coatings, electrical components, bearing and tapes (Stuart, 2002). The PTFE is insoluble in all common solvents and is highly resistant to chemical attack. Its combination of electrical properties is outstanding with high dielectric strength and extremely low dielectric loss. PTFE is very stable over the widest temperature range of any known insulating polymer (Duncan and Mark, 2002). In this paper we report the dielectric properties and AC conductivity of commercial PTFE which is known as Teflon (Trade name), at different temperatures and different frequencies. IR spectra have also been reported.

\section{EXPERIMENTAL METHOD}

\section{A. Sample Preparation}

The PTFE used in the present work is obtained from the commercial source (Nokan Plastic Factory). The PTFE sample is impure and, produced by extrusion molding. For the dielectric measurements, the PTFE sample was cut into slab of $6 \mathrm{~cm}$ in diameter and $1.75 \mathrm{~mm}$ in thickness by using precision micrometer. The surface of the PTFE sample was polished in order to get a smooth surface for good electrical contact between the sample and the electrodes. The dielectric loss cell was locally designed. The heating coil was made from a resistive wire in the form of circular coil arranged symmetrically in the chamber. The resistance coil is connected 
to an A.C source variac transformer. The current through this heater was adjusted to optimal. To eliminate temperature gradient the system was heated for several hours to achieve thermal equilibrium. The sample holders which were used to measure the electric parameters are two identical discs of aluminum $(5.6 \mathrm{~cm}$ diameter). The lower disc is fixed, while the upper is movable by using a screw to ensure good electrical contacts between the electrodes and the sample; this enables us to avoid the parasite capacitance induced by the presence of air interstices at the interfaces between the sample and the electrodes. The thermocouples were used to record the temperature are not attached to the capacitor plates directly because they may produce a leak which may changes the value of the capacitance, therefore it was set as close as possible to the capacitor plate as the thickness of one mica sheet.

\section{B. Infrared (IR) Measurement}

The IR spectra of PTFE polymer was obtained at room temperature in the wave number $(450-4000 \mathrm{~cm}-1)$ with double beam recording IR spectrometer (Perkin Elmer). For IR measurement the solid PTFE was simply converted to powder form (1 mg) using the saw method.
The $\mathrm{KBr}$ and PTFE powder was mixed and then compressed at 10 tones to form $1 \mathrm{~mm}$ thick disk for IR analysis.

\section{Dielectric and AC Measurement}

Dielectric and AC conductivity measurements were performed using Programmable Automatic Precision RCL meter type PM6036. During the experiment, the temperature was measured using Alumel-Chromel constantan thermocouple, which was accurate to $\pm 1^{\circ} \mathrm{C}$, with digital thermometer TM-914C $\left(40 \sim 1200^{\circ} \mathrm{C}\right)$.

The resistance and capacitance of PTFE sample was measured at different temperatures and different frequencies, from which the dielectric constant, dielectric loss and $\mathrm{AC}$ conductivity was calculated by some relations, were presented in later sections.

\section{RESULTS AND DISCUSSION}

\section{A. IR Analysis}

Fig. 1 shows the IR spectra of commercial polytetrafluoroethylene (PTFE) at room temperature.

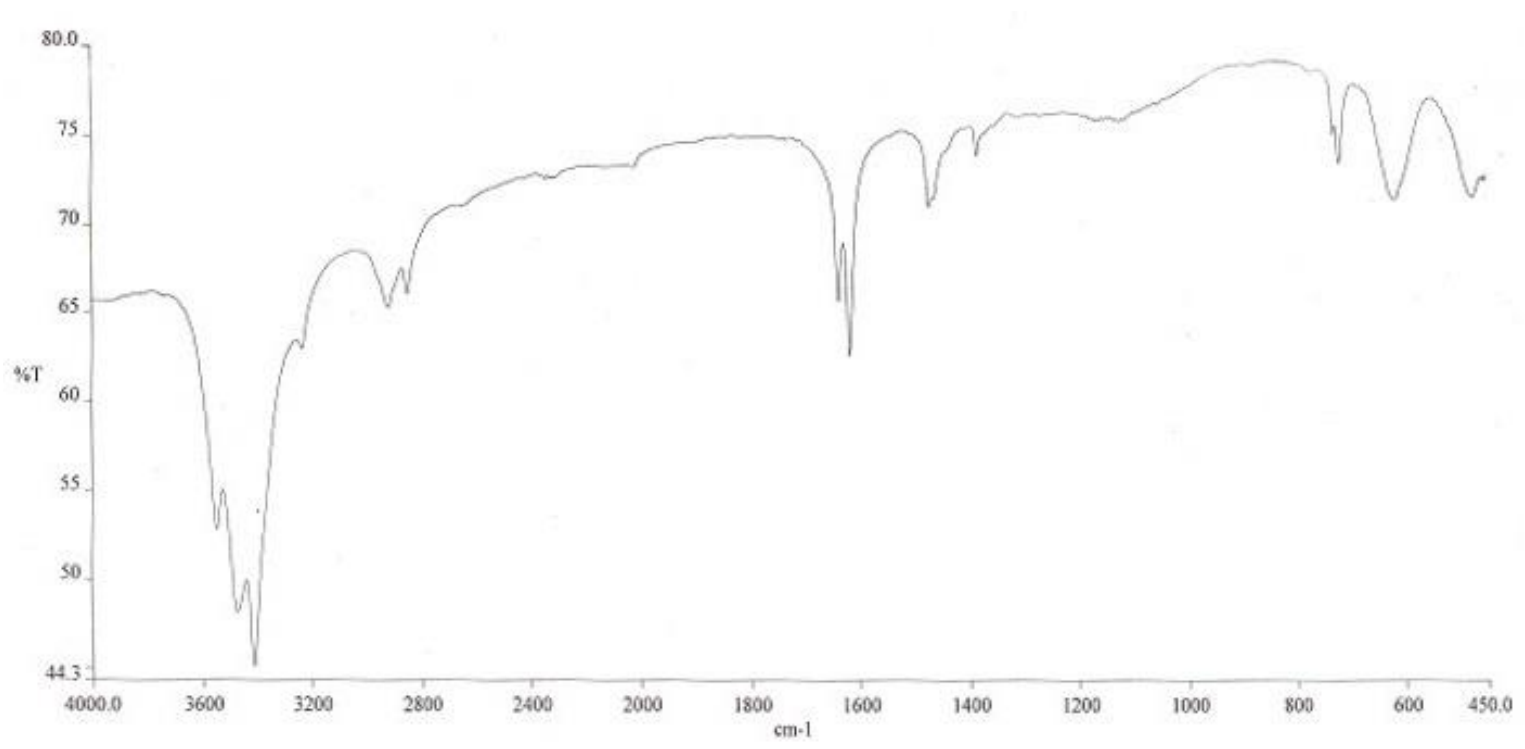

Fig. 1. A photo copy of the measured IR spectra of commercial polytetrafluoroethylene (PTFE) at room temperature.

IR spectroscopy is a popular method for characterizing polymers. This technique is based on the vibrations of the atoms of the molecule (Stuart, 2002). The IR spectra shows a broad band in between 3550 and $2850 \mathrm{~cm}-1$ which can be ascribed to both $\mathrm{OH}$ groups and water absorbed by $\mathrm{KBr}$ (Lucas, 2000; Macipe, et al., 1999). The bands at 1637 and $1617 \mathrm{~cm}-1$ was non-aromatic double bond stretching $\mathrm{C}=\mathrm{C}$. The peak at $1380 \mathrm{~cm}-1$ is attributed to CF bending (Soo-Jin et al., 2003), and the bands at 670 and $595 \mathrm{~cm}-1$ are due to deformation vibrations (F-C-F) of $\mathrm{CF}_{2}$ bending (Yang et al.,
2007). Thus the IR spectrum confirms the existence of unsaturated double bonds in the commercial PTFE sample. The other peaks are due to the impurities exist in the sample especially additives, stabilizers, fillers and many others.

\section{B. Dielectric Analysis}

The study of dielectric relaxation phenomena is a powerful tool for understanding of the ion transport behavior and obtaining the information of ionic and molecular interaction in solid polymer (Pradhan, Choudhary snd Samantaray, 2008). 
The complex permittivity function:

$$
\varepsilon^{*}(\omega)=\varepsilon^{\prime}(\omega)+\varepsilon^{\prime \prime}(\omega)
$$

Which is a materials property depending on frequency of the applied field, where $\varepsilon^{\prime}(\omega)$ a relative permittivity that related to the energy is stored in the material and $\varepsilon^{\prime \prime}(\omega)$ a dielectric loss which is proportional to the energy that is dissipated in each cycle (Okutan and Şentürk, 2008; Batoo, Kumar and Lee, 2009). The dielectric constant, dielectric loss and AC conductivity were estimated using the following relations (Mohamed and Gadou, 2005; Muhammad, Athar and Tasneem, 2005):

$$
\begin{gathered}
\varepsilon^{\prime}=\frac{L \cdot C}{A \cdot \varepsilon_{O}} \\
\varepsilon^{\prime \prime}=\frac{1}{R \cdot C_{o} \cdot \omega} \\
\sigma_{A C}=\frac{L}{R \cdot A}
\end{gathered}
$$

Where $\mathrm{C}$ is capacitance of the polymer sample and:

$$
C_{o}=\frac{\varepsilon_{0} \cdot A}{L}
$$

$\varepsilon_{o}$ is the permittivity of free space $(8.85 \times 10-12 \mathrm{~F} / \mathrm{m})$. A and $\mathrm{L}$ are the active area of the sample and its thickness, respectively, and $R$ is the resistance of the polymer sample. $\omega$ is the angular frequency and is given by; $\omega=2 \pi f$.

The relative permittivity curves of PTFE sample versus frequency at different temperatures are presented in Fig. 2. Dielectric properties are a complex function of bulk permittivity, conductivity, size, shape, spatial arrangement of the constituents (the filler, platicizer and the matrix) and the testing frequency and temperature (Yang, et al., 2007).

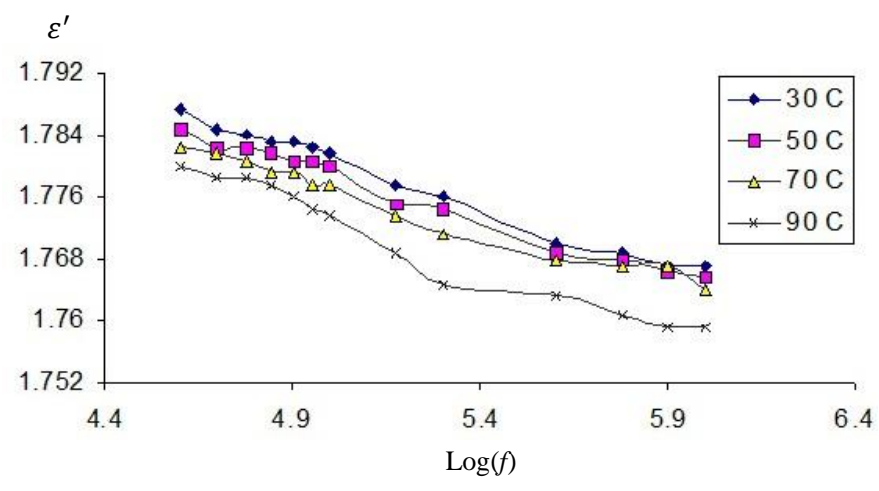

Fig 2. Frequency dependence of relative permittivity $\left(\varepsilon^{\prime}\right)$ at different temperatures for commercial PTFE.

It can be observed that for this nonpolar polymer, the relative permittivity is slightly frequency dependent, with a slight decrease as frequency increases from $10 \mathrm{KHz}$ to $1 \mathrm{MHz}$. There are three types of polarization electronic, ionic and orientation. Dielectric materials ordinarily exhibit at least one of the mentioned polarization types depending on the material and also the manner of the external field application (Kuntaman and Ayten, 2002; Kittel, 2005), polymers such as polystyrene and PTFE, have no polar groups so its permittivity reflects the displacement of electrons relative to nuclei, i. e., electronic polarization when an AC electric field is applied (Kuntaman and Ayten, 2002). The decrease of relative permittivity with increasing frequency due to the dielectric dispersion as a result of the lag of molecules behind the alternation of the electric field at high frequency, i. e., there is no enough time for molecules to follow the alternation of the electric field at high frequency (rapid phase orientation) (Nada et al., 2004). The total variation of relative dielectric constant against frequency and temperature is small, its value ranges between 1.766 and 1.789 and this is due to the nonpolar nature of PTFE and in this polymer the electronic polarization is dominant and occurs during a very short interval of time (of order of 10-15 sec.), (Khare and Sandeep, 2000). In general, the variation of relative permittivity with temperature is different depending on the polarity of the polymers. For nonpolar polymers the relative permittivity is known to be slightly affected by temperature as depicted in Fig. 2 ; but in the case of strong polar polymers is completely different, that is, the relative permittivity increases as the temperature increases (Wong, Youterson and Sutherland, 2005). The dielectric loss versus frequency at different temperatures for commercial PTFE are presented in Fig. 3.

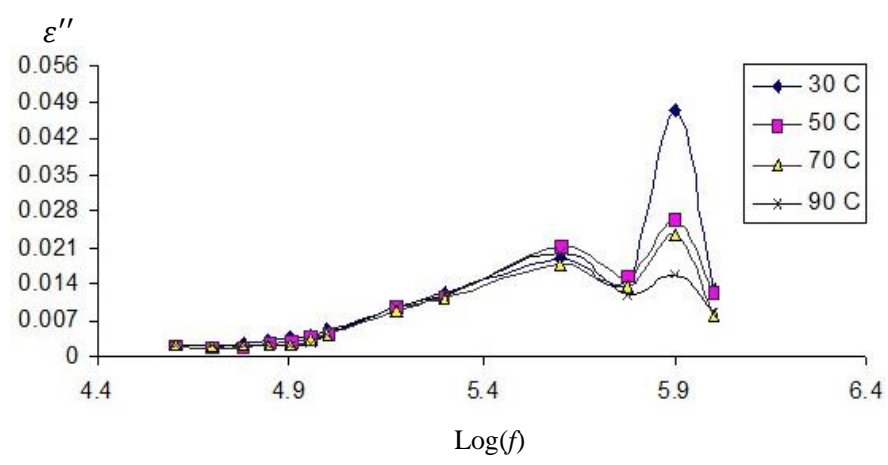

Fig. 3. Frequency dependence of dielectric loss $\left(\varepsilon^{\prime \prime}\right)$ at different temperatures of commercial PTFE

It can be seen that at lower frequencies the dielectric loss (Fig. 3) is small but when the frequency is increased the dielectric loss is increased as well as some loss peaks are appeared which can be attributed to the presence of impurities and carbon double bonds as confirmed in IR study (Fig. 1). The increase of dielectric loss with increasing frequency can be explained as follow: as the applied frequency increases, the inertia of the charged particles tends to prevent the particle displacements from keeping in phase as the field changes. This leads to a frictional damping mechanism that causes power loss, because work must be performed to overcome 
these damping forces. Most dielectrics, including ceramics and polymers, exhibit low loss at low frequencies but become lossy at high frequencies (James, 1998). Low values of $\varepsilon^{\prime \prime}$ (0.0021-0.0473) of PTFE are indicative of minimal conversions of electrical energy to heat and little overall energy loss. Regarding the peaks observed in Fig. 3 which due to the presence of unsaturated double bond $(C=C)$ as confirmed by IR study and polar additives which may introduce dipole moments in PTFE, which may couple the molecular motions of a present sample to the external field and produce the $\gamma$ and $\beta$-dielectric relaxation peaks which shift to high frequency. It can be observed that the peaks are broad and they are not symmetrical about its maximum, this suggests the fact that these peaks are due to distributed relaxation.

The dielectric loss versus relative permittivity (Cole-Cole plot) at temperatures $30{ }^{\circ} \mathrm{C}$ and $70{ }^{\circ} \mathrm{C}$ are presented in Fig. 4 and Fig. 5. The shapes of the curves indicate that there are two different relaxations are contributing to the relaxation data. The Cole-Cole plots shows a broad dispersion for $\varepsilon^{\prime \prime}-\varepsilon^{\prime}$ curve, which is correspond to a distribution of relaxation time and we have a lower loss maxima than those predicted by the Debye model and the $\varepsilon^{\prime \prime}-\varepsilon^{\prime}$ curves not falls inside the semicircle. The Cole-Cole plots from 30 to $70{ }^{\circ} \mathrm{C}$ inform us that the relaxation even in this temperature range cannot be described by single Debye relaxation. According to Debye model the $\varepsilon^{\prime \prime}$ versus $\varepsilon^{\prime}$ must give a semicircle with a diameter coincides with the $\mathrm{x}$ axis. Thus a non-Debye type relaxation can be concluded in commercial PTFE sample. In solid polymers, the dielectric response departs noticeably from the Debye behavior. The full width at half maximum (FWHM) is 0.007 and 0.008 at temperatures $30^{\circ} \mathrm{C}$ and $70^{\circ} \mathrm{C}$ respectively. Thus the FWHM of dielectric loss peaks are significantly smaller than 1.144 decades as predicted by the Debye model. In many cases the loss peaks are also observed to be asymmetric about the maximum peak frequency $f_{\mathrm{p}}$ (Dilip, 1994), as it can be seen in the present work (Fig. 4 and Fig. 5).

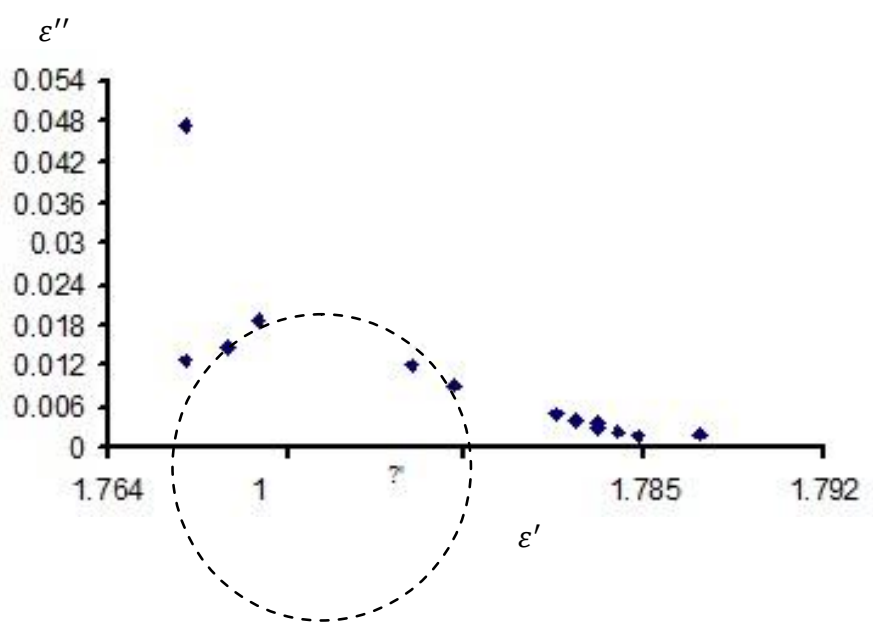

Fig.4. Cole-Cole ( $\varepsilon^{\prime \prime}$ versus $\left.\varepsilon^{\prime}\right)$ plot at $30^{\circ} \mathrm{C}$.

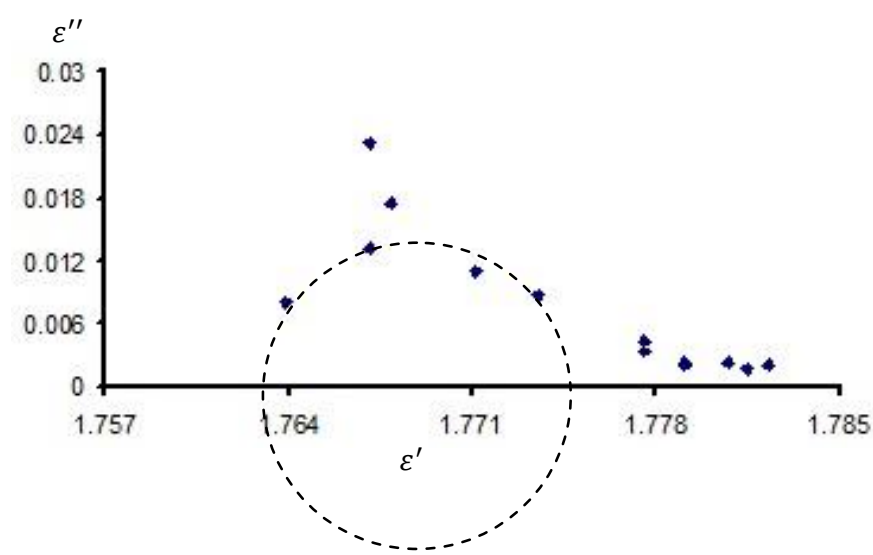

Fig.5. Cole-Cole $\left(\varepsilon^{\prime \prime}\right.$ versus $\left.\varepsilon^{\prime}\right)$ plot at $70^{\circ} \mathrm{C}$.

\section{The AC Conductivity Analysis}

Fig. 6 shows the frequency dependence of AC conductivity at different temperatures.

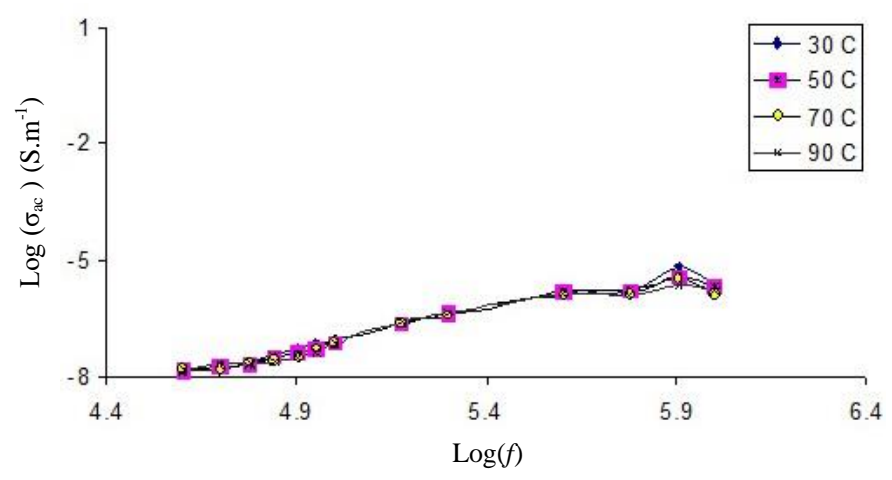

Fig. 6. Frequency dependence of AC conductivity at different temperatures of commercial PTFE.

It is clear that the $\mathrm{AC}$ conductivity increase with increasing of frequency almost linearly. The deviation from the linearity at higher frequencies is ascribed to dispersion of molecules. The increase in AC conductivity with frequency and weak temperature dependence indicate that charge carriers are transported by hopping processes through defect sites along the chain (Vijayalakshmi, Ashokan and Shridhar, 2000). The variation of $\mathrm{AC}$ conductivity with temperature at different frequencies is presented in Fig. 7.

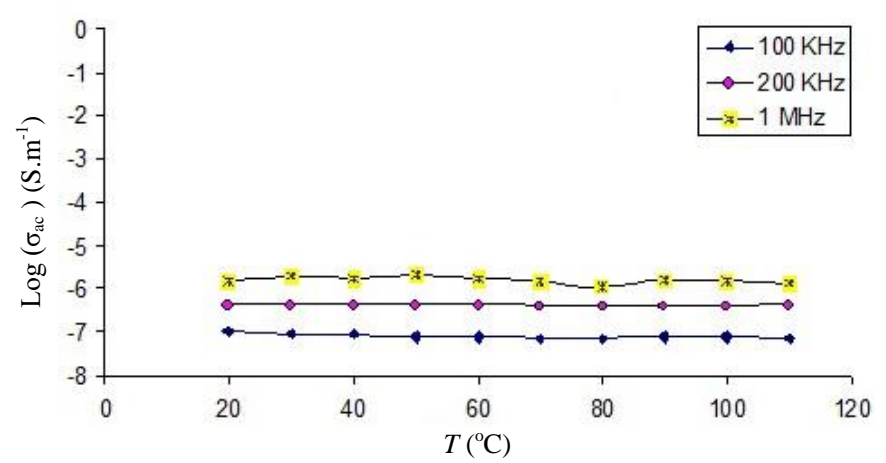

Fig. 7. Temperature dependence of AC conductivity at different frequency of commercial PTFE. 
As it is clear from Fig. 7 that the AC conductivity is almost temperature independent, but it can be observed that the $\mathrm{AC}$ conductivity increases with increasing frequency and has the higher value at $1 \mathrm{MHz}$. This is due to frequency dependent of AC conductivity $\left(\sigma_{\mathrm{AC}}=2 . \pi \cdot f \cdot \varepsilon_{0} \cdot \varepsilon^{\prime \prime}\right)$ (Tony and David, 2005; Saravanan, Anantharamanand and Venkatachalam, 2006).

\section{CONCLUSION}

The relative permittivity decreases with increasing frequency which can be attributed to the occurrence of dielectric dispersion, while its decrease with temperature can be ascribed to the temperature dependence of specific volume of the commercial PTFE sample. The appearance of some peaks in dielectric loss spectra are due to unsaturated double bonds $(C=C)$ and many other impurities, which indicating the dielectric relaxation phenomena. From the $\varepsilon^{\prime \prime}$ spectra measurement we conclude that the commercial PTFE is a low loss material. The uncompleted semicircle between $\varepsilon^{\prime}$ and $\varepsilon^{\prime \prime}$ and the calculated value of FWHM at different temperatures reveal the non-Debye relaxation processes in PTFE sample. The increase of AC conductivity with increasing frequency is due to charge carrier's conduction via hopping process.

\section{ACKNOWLEDGMENT}

We are grateful to thank Dr. Emad A. Al-Khafagy for IR analysis. Great thanks due to Salaadin University, Physics department, for LCR meter equipment.

\section{REFERENCES}

Batoo, K.M., Kumar. S. and Lee C.G., 2009. Study of dielectric and ac impedance properties of Ti doped Mn ferrites. Current Applied Physics, 9(6), pp.1397-1406.

Dilip, K.D., 1994. Polyethylene: Structure, Morphology, Molecular Motion and Dielectric Behavior. IEEE Electrical Insulation Magazine, 10(3), pp.5-15.

Duncan, M.P., and Mark, J., 2002. Thermal conductivity of PTFE and PTFE composites. Thermochimica Acta 392-393, pp.231-236.

Fujii, M., Kazuyuki, T., Tokoro T. and Mizuno, Y., 2006. Development of new Electrode system for High Field Dielectric Properties Measurement Using Evaporated PP Thin guard Film. IEEJ Transcations on Funadamentals and Materials, 126(7), pp.716-722.

James, R.W., 1998. Thin Film Polymer Dielectrics for High-Voltage Applications under Severe Enviroments. M.Sc. Thesis, Virginia Polytechnic Institute and State University.

Khare, P.K., and Sandeep, K.J., 2000. Dielectric properties of solution-grown- undoped and acrylic-acid-doped ethyl cellulose. Bulletin Materials Science, 23(1), pp.17-21.

Kittel, C., 2005. Introduction to solid state physics. $8^{\text {th }}$ Edition, John Wiley \& Sons.

Kuntaman, H. and Ayten, K., 2002. A study on dielectric modeling of a new synthesized polyimide. Journal of Electrical and Electronics, 2(1), pp.359367.

Lucas, M.H., 2000. Transparent and conductive polymer layers by gas plasma techniques. Ph.D Thesis, Twente Universiteit.

Macipe, A.L., Burgues, J.T., Morales, J.G. and Clemente R.R., 1999. Continuous precipitation of hydrixyapatite from $\mathrm{Ca} /$ citrate/phosphate solutions using microwave heating. Crystal Research and Technology, 34(6), pp.757-762.

Mohamed, R.I., and Gadou, A.M., 2000. AC-Conductivity and dielectric properties of $\gamma$-Irradiated PVA films doped with Mn2+ Ions. Egypt J. Sol., 23(1), pp.277-286.

Muhammad, A., Athar, J. and Tasneem, Z.R., 2005. Dielectric Properties of Industrial Polymer Composite Materials. Turk J Phys, 29, pp.355-362.

Nada, A.M.A., Dawy M., and Salama A.H., 2004. Dielectric properties and ac-conductivity of cellulose polyethylene glycol blends. Materials chemistry and Physics, 84, pp.205-215.

Okutan, M. and Şentürk, E., 2008. $\beta$ Dielectric relaxation mode in side-chain liquid crystalline polymer film title am papera bdozarawa. Journal of NonCrystalline Solids, 354, pp.1526-1530.

Pradhan, D.K., Choudhary, N.P. and Samantaray, B.K., 2008. Studies of Dielectric Relaxation and AC Conductivity Behavior of Plasticized Polymer Nanocomposite Electrolytes. International journal of Electrochemical Science, 3, pp.597-608.

Saravanan, S., Anantharaman, M.R.M. and Venkatachalam, S., 2006. Structural and electrical studies on tetrameric cobalt phthalocyanine composites. Materials Science and Engineering. B, 135, pp.113-119.

Singh V., 2006. Physico-chemical studies of swift heavy ion modified polymers. Ph.D Thesis, Panjab University, Chandigarh.

Soo-Jin, P., Su-Wan, S., Min-Kang, S., Jae-Sup S., and Kyuchul, K., 2003. A study on PTC/NTC Behaviour of Fluorinated Carbon Black-Filled HDPE Matrix Compounds. Journal of the Korean Society, 47(2), pp.147-154.

Stuart, B.H., 2002. Polymer Analysis, John Wiley \&Sons, Ltd.

Tony, B. and David, B., 2005. Electrical Properties of Polymers" Cambridge University Press, $2^{\text {nd }}$ Edition.

Vijayalakshmi, R., Ashokan, P.V., Shridhar, M.H., 2000. Studies of dielectric relaxation and a.c. conductivity in cellulose acetate hydrogen phthalate-poly (methyl methacrylate) blends. Materials Science and Engineering A281, pp.213-220.

Wong, S., Youterson, E. and Sutherland, E., 2006. Dielectric Properties of graphite nanocomposites. Journal of Vinyl and Additive Technology, 12, pp.127-130.

Yang, S., Benitez, R., Fuentes, A. and Lozano, K., 2007. Dielectric analysis of VGCNF reinforced polyethylene composites. Composite Science and Technology, 67, pp.1159-1166. 